\title{
Diseño y ejecución de un curso de pregrado sobre subtitulación
}

\author{
Design and Execution of an Undergraduate Subtitling Course \\ Planejamento e execução de um curso de ensino superior sobre subtitulação
}

Iván Alejandro Villanueva Jordán*

Recibido: 18/02/16

Facultad de Ciencias Humanas - Universidad Peruana de Ciencias Aplicadas, Lima, Perú

Aceptado: 19/05/16

RESUMEN. El artículo presenta el perfil curricular de un curso introductorio a la subtitulación en un programa universitario de traducción en Lima, Perú. Con este fin se documentan los componentes del diseño que conllevaron su ejecución: los contenidos por unidad, la metodología y la evaluación. Se espera que el artículo evidencie los puntos clave del planeamiento y promueva el ámbito aplicado de la didáctica de la traducción audiovisual. El artículo presenta el perfil curricular de un curso introductorio a la subtitulación en un programa universitario de traducción en Lima, Perú. ${ }^{1}$
Palabras clave: subtitulación, traducción audiovisual, didáctica de la traducción, competencia traductora

\begin{abstract}
This paper presents the curricular profile of an introductory course to subtitling in a university translation program in Lima, Peru. To this aim, the design elements that lead to its implementation are documented: contents per unit, methodology and evaluation. The paper is expected to evidence the key points of the proposal and to promote the practical application of audiovisual translation didactics.
\end{abstract}

Keywords: subtitling, audiovisual translation, translation didactics, translation competence
RESUMO. O artigo apresenta o perfil curricular de um curso de introdução a subtitulação em um programa universitário de tradução em Lima, Peru. Com este objetivo, justificasse os componentes do projeto que fizeram parte da sua execução: os conteúdos por unidade, a metodologia e a avaliação. 0 artigo destacará os pontos chaves do planejamento e mencionará o âmbito aplicado da didática da tradução audiovisual.
Palavras chave: subtitulação, tradução audiovisual, didática da tradução, competência tradutora

Citar como: Villanueva, I. (2016). Diseño y ejecución de un curso de pregrado sobre subtitulación. Revista Digital de Investigación en Docencia Universitaria, 10(1), 52-67. doi: http://dx.doi.org/10.19083/ridu.10.460

*E-mail: ivan.villanuevalupc.edu.pe 
Si bien la subtitulación es una práctica de mediación interlingüística que ha acompañado el desarrollo de los medios audiovisuales desde las primeras décadas del siglo XX, la oferta de especialización en este campo parece haber surgido de manera paralela a la concretización del interés de la Traductología en esta práctica durante la década de 1990 (Martínez Sierra, 2012al. Desde entonces, la cantidad de programas de especialización en traducción audiovisual, tanto a nivel de pregrado como de posgrado, ha crecido exponencialmente en distintos países de Europa, como España, Italia, Francia e Inglaterra (Cerezo, 2013). Sin embargo, este no ha sido el caso en el Perú donde, solo ahora, las ofertas de formación universitaria han comenzado a incluir cursos de traducción audiovisual con fines de inducción o de especialización.

El objetivo general de este artículo es describir el diseño y abordar con detalle los componentes curriculares del curso Doblaje y Subtitulación (TR160) del programa de Traducción e Interpretación Profesional de la Universidad Peruana de Ciencias Aplicadas (TIP-UPC). Se busca alcanzar este objetivo desde una perspectiva curricular y pedagógica de la traducción audiovisual, y mediante la recopilación de la información instructiva que enmarca dicho curso y la percepción docente de los implicados en su diseño y ejecución.

Este artículo se inicia con una breve reflexión sobre el estado actual de los servicios especializados de subtitulación interlingüística en el medio local (Lima, Perúl que, a suvez, conllevó el razonamiento de base para incluir un curso introductorio a la traducción audiovisual en un programa de formación profesional de pregrado. A continuación se presenta, brevemente, el programa TIP-UPC con un enfoque particular en su modelo por competencias y la competencia traductora prospectiva desarrollada para la articulación de los distintos cursos y talleres de traducción. En la tercera sección, la de mayor extensión, se plantean los componentes básicos del diseño del curso TR160 y se ponen de relieve las dos primeras unidades temáticas de dicho curso, vinculadas ambas con la subtitulación. El trabajo culmina con algunas ideas puntuales sobre aspectos que deben mejorarse en las siguientes ediciones del curso, así como la valoración positiva de aquellos componentes que tuvieron los mejores resultados.
Se trata así de un artículo de estructura similar a los trabajos publicados por Granell (2011) y Díaz-Cintas y Orero (2003) para el course profiling de un taller de localización de videojuegos y un programa de maestría en traducción audiovisual, respectivamente. Está claro que, debido al carácter inicial de esta propuesta curricular, los alcances que se brinden en el artículo son modestos en relación con los de los autores mencionados.

\section{BREVES COMENTARIOS SOBRE LA TRADUCCIÓN AUDIOVISUAL EN EL PERÚ}

En el ámbito académico de la traducción, la etiqueta traducción audiovisual se utiliza para denominar una serie de modalidades de traducción que hacen accesibles contenidos audiovisuales que, de otra forma, no podrían ser consumidos por razón de desconocimiento lingüístico e inclusive por razón de discapacidad auditiva o visual. Como sucede en el ámbito internacional, en el Perú esta denominación parte de la academia y coexiste con otras formas en las que los profesionales de los medios de comunicación denominan los procesos de posproducción, como la subtitulación y el doblaje. Cabe señalar en este punto que la traducción, como un ámbito de especialidad en el Perú, es poco conocida. Existe la confusión usual entre el perfil del lingüista, el traductor, el intérprete o un sujeto políglota sin credenciales académicas. Este desconocimiento se expande al ámbito de los productos audiovisuales mediados; suelen oírse frases como «la película traducida». Así, el uso de los términos subtitulación o doblaje queda restringidos solo al público especialista en traducción, al cine o la comunicación audiovisual.

La subtitulación, como una modalidad de traducción audiovisual, existe plenamente en el Perú debido al consumo generalizado de productos audiovisuales en lenguas extranjeras (en particular el cine, y también otros medios de comunicación). A pesar de la cantidad de producciones cinematográficas extranjeras que se exhiben en el Perú y los distintos géneros fílmicos, no podría ser categorizado exclusivamente como un país doblador o subtitulador. Los medios de comunicación locales de señal abierta solo transmiten productos audiovisuales doblados; no sucede así en el caso de los canales de televisión local de pago, que incluyen en su programación productos subtitulados. 
Las salas de cine comercial, como se mencionó antes, exhiben películas dobladas y subtituladas; la modalidad se vincula lógicamente con el género fílmico y los destinatarios objetivos. Sin embargo, se podrían revelar algunas tendencias en la cantidad de películas dobladas frente al número de películas subtituladas en determinados distritos de Lima; los centros más urbanizados contarían así con mayor oferta de películas subtituladas, mientras que las comunas en proceso de desarrollo o expansión, con una oferta restringida al doblaje. Asimismo, a partir de un contraste de las carteleras publicadas en un conocido diario peruano, existen algunas restricciones en las salas de cine de provincias, en las que solo se suele contar con versiones dobladas - posiblemente, con el fin de reducir los costos de exhibiciones diferenciadas por la modalidad de traducción- (El Comercio, 2014).

Estas últimas ideas en torno a las exhibiciones comerciales dan una visión general de la visibilidad de la traducción audiovisual en el Perú. Como se dijo anteriormente, la subtitulación y el doblaje coexisten; sin embargo, su práctica profesional es desconocida por el público no especializado. En la subsección siguiente se aborda el tema del cine no comercial y la manera en que los servicios especializados de traducción audiovisual no se encuentran localizados en el gremio de traductores. La razón propuesta: una diáspora de encargos hacia profesionales de otros ámbitos debido a la falta de competencias específicas.

\section{LOS SERVICIOS ESPECIALIZADOS DE SUBTITULACIÓN}

La subtitulación también es una realidad en el Perú en el marco del cine no comercial, de exhibiciones enfocadas en la difusión artística, la interculturalidad y la puesta de relieve de las minorías. Esto se evidencia en los distintos festivales de cine que se llevan a cabo en todo el país². Las dinámicas propias de estos eventos que reúnen productos y productores de distintos espacios, con distintas lenguas y agendas, conducen necesariamente a encargos de traducción, en muchos casos, con alta premura, poco presupuesto y pocos recursos de base-como guiones de posproducción o transcripciones inexistentes-. A pesar de estas condiciones, la pregunta sobre quién se dedica a traducir estos productos sigue siendo pertinente.
La subtitulación también existe en los entornos institucionales privados, en los que se producen videos de difusión interna de corte instructivo o promocional; estos audiovisuales pueden verse en empresas privadas, salas de estar, en las pequeñas pantallas incrustadas en los asientos de avión, entre otros. Un ejemplo paradigmático de este entorno de subtitulación es el de la campaña nacional Marca Perú. Este producto se exhibe a nivel nacional e internacional, en diversos espacios de instituciones vinculadas con la inversión privada extranjera ${ }^{3}$. A su vez, en este documental también resulta evidente la falta de criterios que existe en el tratamiento de la mediación interlingüística de audiovisuales. ¿Quién subtitula este tipo de productos?

Los encargos de traducción audiovisual parecen estar destinados a los profesionales de la comunicación audiovisual, empresas de realización audiovisual, personas bilingües con conocimiento de edición de videos, inclusive estudiantes universitarios de pregrado que toman los encargos como prácticas preprofesionales o una salida profesional alterna. Este tipo de servicios de subtitulación encajaría en lo que se puede denominar «oferta generalista», es decir, los servicios de subtitulación ofertados que no consideran la criterios de calidad fundamentales de la subtitulación interlingüística.

Lo interesante de pensar en la subtitulación en el Perú es notar que existe una cantidad de encargos que suceden a nivel local a pesar de que las ofertas de formación en traducción audiovisual son bastante recientes. Existe una demanda real de subtitulación, así como una oferta de servicios que no consideran estándares de calidad en la medida que los traductores, como especialistas, no pueden ofrecer una oferta variada o diferenciada de aquella del traductor general o del profesional de otros ámbitos. Esto implica, en la dinámica de mejora de los servicios y de la competitividad en el mercado, que se ingresa a un círculo de oferta y demanda que carece de innovación y mucho más de estándares de calidad básicos.

Una oferta generalista no conduce a cambios en las dinámicas de trabajo no especializado, ya que los usuarios de los servicios han recibido comúnmente 
productos de una calidad inferior a la de los estándares extranjeros. En la medida que se pueda pensar en un influjo de servicios especializados, se podrá ofrecer un mejor servicio, más competitivo, con mejores criterios de calidad. En este punto se asume como una necesidad pensar en ofertas de formación especializada en traducción audiovisual; este déficit real en el mercado de la traducción en Perú es, como propone Kelly (2005) en su diagrama para el planeamiento instruccional, la conclusión del análisis del contexto institucional y social. Ello permite ahora proponer ideas sobre la formación especializada en traducción audiovisual.

\section{LA FORMACIÓN EN TRADUCCIÓN AUDIOVISUAL EN EL PERÚ}

La falta de oferta especializada de subtitulación se debe, sin duda, a la falta de formación especializada. Sin embargo, se podría aducir que la falta de formación especializada es porque no hay una demanda de este tipo de servicios de traducción. Este planteamiento cada vez resulta menos convincente: en el Perú sí se subtitula. Otra podría ser la razón para la virtual inexistencia de encargos de traducción audiovisual. Este tipo de encargos se han derivado a otro tipo de profesionales. Sin embargo, no se trata de oportunidades arrebatadas, sino de una falta de oferta de servicios de traductores especializados. El desconocimiento por parte de los propios profesionales de la traducción para enfrentarse a este tipo de encargos termina en que sean otros los que deban asumir estas actividades.

Sería injusto plantear que no se han hecho esfuerzos para la formación de traductores audiovisuales en el Perú. Se han brindado cursos cortos vinculados con la subtitulación y el doblaje en los últimos años; muchos de estos por parte de empresas de traducción que además cuentan con áreas de formación profesional continua, así como de oficinas de extensión profesional de las universidades con ofertas de formación de traductores e intérpretes. Se puede mencionar como un giro en este tipo de ofertas no institucionalizadas o sin creditaje académico el programa de posgrado en Traducción de la Universidad Ricardo Palma, que incluye el curso de Doblaje y Subtitulación (de 20 horas de trabajo presencial y tiempo fuera de aula para la preparación de proyectos) y que ha tenido dos ediciones, el 2013 y el 2014.

El curso de Doblaje y Subtitulación (TR160) del programa de Traducción e Interpretación Profesional de la Universidad Peruana de Ciencias Aplicadas (TIP-UPC) comenzó a dictarse en marzo de 2015. La justificación de un curso obligatorio de esta temática surge del interés en promover nuevas salidas profesionales para los traductores en proceso de formación. Se trata del primer curso de pregrado de este tipo y su planeamiento y ejecución requirieron de un amplio trabajo de investigación y del esfuerzo conjunto de los docentes a cargo de su dictado para proponer contenidos, elaborar material didáctico y enfrentar distintas contingencias durante el desarrollo del curso. En lo que sigue se plantean algunas características del programa en general y otros componentes de este curso introductorio.

\section{MODELO DE COMPETENCIA DE TRADUCTOLOGÍA TIP-UPC}

La carrera TIP-UPC inició sus actividades en 2011 con la primera convocatoria de ingreso semestral. El programa cuenta con diez niveles, cada uno desarrollado durante un cuatrimestre. Ello implica una formación de pregrado de cinco años, que busca equilibrar el influjo de carácter profesional que contribuye a la empleabilidad de los egresados y el carácter académico que caracteriza la formación universitaria en el ámbito de las humanidades. Los egresados de la carrera obtienen el grado de Bachiller en Traducción e Interpretación Profesional y luego pueden optar por el título profesional de Licenciado en Traducción e Interpretación Profesional ${ }^{4}$. El programa ofrece como lenguas de especialidad el inglés (de forma obligatorial y el chino, francés, portugués o quechua (como lenguas optativas).

TIP-UPC es un programa académico basado en competencias, tanto generales (relacionadas con el modelo educativo de toda la universidad) como específicas (relacionadas con el perfil específico del egresado de la carreral ${ }^{5}$. El programa se basa en un total de ocho competencias específicas que son las siguientes: Habilidades Interpersonales, Interculturalidad, Interpretación, Investigación, Lengua Materna, Segundas Lenguas, Tecnología y Traductología. 
El desarrollo y adquisición de estas competencias está garantizado mediante los distintos cursos y talleres de la carrera, cada uno de los que contribuye de forma efectiva, secuencial y dosificada con los distintos criterios y niveles de competencia propuestos.

La competencia de Traductología desarrolla de manera directa las que se conciben como habilidades específicas del traductor ${ }^{6}$. Cabe resaltar que este modelo de competencia traductora no se basa en una propuesta descriptiva del perfil profesional local, sino en una propuesta prospectiva y generadora de cambio que se estableció durante los primeros pasos del planeamiento del programa ${ }^{7}$. La competencia de Traductología tiene cuatro niveles de desarrollo, así como cuatro dimensiones (o subcompetencias) que colaboran con el mapeo de las habilidades a lo largo del programa profesional, en cada nivel de la carrera.

Por ejemplo, en el caso del curso de Doblaje y Subtitulación (TR160) que se presenta en este artículo, las coordenadas de la competencia traductora incluyen los siguientes descriptores.

Como se aprecia en la Tabla 1, las dimensiones (1 al 4) de la fila superior son desagregados de la competencia de Traductología; en ese sentido se parte de una concepción componencial de la propia competencia traductora. Por otro lado, los niveles de logro de la competencia varían de acuerdo con los contenidos del curso y al grado de especialidad que requieran las tareas y actividades propias del diseño. Si bien este curso se encuentra en el noveno ciclo del programa, los descriptores de la competencia de Traductología plantean que el curso podría ubicarse en niveles inferiores, como el séptimo u octavo nivel. Este tipo de mapeo de los niveles de competencia resulta valioso para las reformulaciones curriculares de un programa profesional.

\section{CURSO DE DOBLAJE Y SUBTITULACIÓN (TR160) EN EL PROGRAMA TIP-UPC}

La primera edición de un curso o asignatura implica un diseño básico que permita el desarrollo temático, así como la práctica y la evaluación de competencias. Es a partir de los niveles de logro que los estudiantes demuestren al finalizar los ciclos académicos que se pueden realizar modificaciones o se pueden establecer las actividades y el material que mejor haya funcionado para la elaboración de carpetas pedagógicas. En esta línea de pensamiento, los postulados de Kelly resultan importantes para las siguientes adecuaciones del diseño de este curso; «this means that it is essential for teachers to be aware of the make-up of each student group and how it may influence class activities» (Kelly,

Tabla 1

Ejemplo de dimensiones y niveles de la competencia específica de Traductología TIP-UPC

\section{DIMENSIÓN 1: Habilidad metatraslativa}

\section{Nivel 3}

Elabora planteamientos traductológicos sobre

la base de su propia experiencia y relacionados con modelos teóricos.

\section{DIMENSIÓN 2: Habilidad traslativo-estratégica}

\section{Nivel 2}

Traduce de manera directa tomando en cuenta los componentes del encargo de traducción, así como las características lingüísticas y pragmáticas del texto fuente.
DIMENSIÓN 3:
Habilidad
instrumental-cualitativa

Nivel 3

Utiliza programas informáticos ad hoc para

realizar encargos de traducción audiovisual.

\section{DIMENSIÓN 4: Habilidad ético-profesional}

Nivel 3

Se desempeña en distintos roles del proceso de traducción en el marco de situaciones dirigidas que requieren de criterio ético.

Nota: A partir del 2016, la competencia especifica incluye una quinta dimensión de productividad que abarca la cantidad de palabras que los estudiantes deben ser capaces de traducir, editar y revisar por hora. 
2005, p. 46). Inclusive, durante el desarrollo de la asignatura - durante su primera edición o cuando ya se haya dictado por algunos semestres-, se pueden realizar cambios en el plan general, en las actividades de práctica o evaluación, para así garantizar el alcance de los logros de aprendizaje.

Con base en esta concepción del diseño de una asignatura es que se presentan los componentes básicos del curso TR160. Se trata de una propuesta que ha sido evaluada luego de su primera ejecución y por los resultados satisfactorios que se obtuvieron mediante las distintas evaluaciones y la propia retroalimentación de los alumnos sobre el desarrollo del curso. Cabe resaltar que solo se abordarán los componentes de la primera mitad del curso dedicados a la subtitulación interlingüística.

Sumilla. La sumilla general del curso se centra en los componentes traductológicos fundamentales que guiaron los siguientes desarrollos del contenido.

Doblaje y Subtitulación es un curso especializado que pertenece a la línea curricular de Traductología. El curso aborda la traducción audiovisual interlingüística en las modalidades del doblaje y la subtitulación, con énfasis tanto en la faceta procesual como en la del producto. En este sentido y desde un enfoque tripartito (funcional, normativo y semiótico) se tratan las principales convenciones y problemáticas de ambas modalidades. Por ello, el curso tiene un carácter multitextual que promueve tanto el desarrollo de estrategias de traducción pertinentes como el dominio de programas informáticos ad hoc.

En esta sumilla se especifica el carácter interlingüístico de la subtitulación y el doblaje para delimitar que se trabajaría siempre con textos en una lengua extranjera que deben traducirse y adaptarse a la lengua meta. En todo caso, el curso ha contemplado solo la traducción directa. El carácter procesual de ambas modalidades de traducción audiovisual se pone de relieve no por las etapas de posproducción de los audiovisuales, sino por el proceso de resolución de problemáticas en el que se enfocan todos los talleres de traducción del programa. En el caso de la mención del producto de traducción, se busca evidenciar que también existe cuidado con la evaluación y control de calidad de los subtítulos y diálogos para el doblaje que los estudiantes producen.

Los tres enfoques que se mencionan son también comunes para los distintos talleres de traducción del programa. El enfoque funcionalista tiene cabida debido a la concepción de que las traducciones se realizan con base en necesidades puntuales por parte de un usuario (Nord, 2005, 2014); los conceptos de corte teórico de este enfoque se concretizan en encargos de traducción máximamente reales con el fin de motivar a los estudiantes, brindarle coherencia a las tareas y establecer criterios de corrección de los productos. En el caso de lo normativo, se toman en cuenta las propuestas descriptivas de la traducción y, en particular, la categoría de normas operacionales que podrían ejemplificarse, claramente, en los protocolos de subtitulación y la necesidad de adecuarse a estos (Díaz-Cintas \& Remael, 2007; Toury, 2004). Finalmente, el enfoque semiótico se refiere tanto a los componentes del texto audiovisual como a las distintas unidades con carga cultural, por ejemplo, que puedan hallarse en los segmentos lingüísticos.

El carácter multitextual es otra característica propia de los talleres de traducción; esto quiere decir que los textos que se traducen no se encuentran restringidos por un tipo de género o campo de especialidad. Esta característica es más relevante en las unidades dedicadas al doblaje, en las que se recurre a distintos géneros audiovisuales. En el caso de las unidades dedicadas a la subtitulación, hubo una marcada preferencia por los productos cinematográficos; esta decisión se explicará más adelante.

Logros. Debido al enfoque pedagógico del programa, la denominación de los resultados de aprendizaje, evidenciados en las aptitudes y habilidades de los estudiantes para la ejecución de distintas tareas, es la de «logro». El curso TR160 cuenta con el siguiente logro general:

El alumno produce diálogos para el doblaje, así como subtítulos en la lengua meta adecuados a las convenciones de la traducción audiovisual, las características del texto audiovisual y el encargo de traducción. 
Por otro lado, los logros específicos de las dos primeras unidades son los siguientes:

a) Analiza secuencias o extractos de productos audiovisuales tomando en cuenta las características semióticas de la traducción audiovisual.

(b)Subtitula secuencias de filmes mediante el uso de programas informáticos y la resolución de problemáticas específicas.

Como se mencionó anteriormente, existe un influjo de la perspectiva descriptivista y funcionalista de la traducción en el diseño del curso. Por ello, el logro general incluye referencias a la adecuación a las convenciones propias de la subtitulación y el doblaje, y al encargo de traducción. En el caso del doblaje, se hace énfasis en la producción de diálogos para el doblaje interlingüístico, lo que determina el alcance de las actividades y los contenidos para la producción de traducciones verosímiles y que cumplan con los criterios de sincronía esperados.

Por su parte, el logro específico de la primera unidad es una evidencia del entrenamiento que se planteó para que los estudiantes se enfrenten al texto audiovisual; en ese sentido y como se verá en la siguiente sección, se promovió el desarrollo de habilidades analíticas de los componentes cinematográficos, en materia técnica y en materia diegética. La segunda unidad, a su vez, se centró en las operaciones básicas para la subtitulación: traducción, localización, segmentación, adaptación y criterios de control de calidad.

Tabla 2

Secuenciación de temas de la primera unidad
Unidades y contenidos. El curso se divide en cuatro unidades, dos previas a la evaluación parcial y dos posteriores. La primera unidad (ver Tabla 2) presenta el concepto de la traducción audiovisual desarrollado en el marco de la Traductología, así como una breve introducción teórico-operativa al texto audiovisual y a la subtitulación. Se explora cuál es la posición de la traducción audiovisual mediante una revisión del surgimiento de esta necesidad lingüística en los filmes hablados y los siguientes desarrollos que la convirtieron en una práctica cotidiana imbricada a las industrias de contenido, las nuevas tecnologías y los ámbitos de especialidad, como la Traductología. Asimismo, esta unidad presenta el contenido conceptual necesario para que el estudiante comprenda los productos audiovisuales como textos y así pueda desarrollar análisis enfocados en las posibles problemáticas de traducción que surjan del ejercicio intersemiótico. Esta primera unidad se halla fundamentada en la propuesta de Lachat (2011) y lo que ella denomina «enseñar a mirar». Asimismo, cabe mencionar el interés de Remael (2004) en el desarrollo de habilidades interpretativas de los diálogos de las películas y, claro está, la manera en que estos colaboran con la narrativa. Hay que remarcar que los contenidos de orden semiótico que se planteaban en la sumilla del curso hallan su nicho en esta primera unidad. Por su carácter introductorio, esta unidad aborda, en su última sección, los contenidos que permitirán un ingreso directo al ejercicio de la subtitulación durante la segunda unidad.
NÚMERO DE SESIÓN

\section{TEMAS DE LA SESIÓN}

Breve historia de la traducción audiovisual en el cine

Aspectos traductológicos de la traducción audiovisual

Conceptos cinematográficos aplicados al análisis de escenas

Elaboración de sinopsis y análisis técnico y diegético

Carácter teórico, normativo y profesional de la subtitulación

Presentación de programas informáticos para la subtitulaciòn 
La segunda unidad (ver Tabla 3) se dedica exclusivamente a la subtitulación desde el enfoque tripartito antes mencionadoy mediante el uso de programas informáticos de licencia libre para el ajuste de los subtítulos. Es una unidad de tipo aplicativo, puesto que se basa en los contenidos conceptuales presentados durante las tres primeras semanas del curso, así como en los análisis que el alumno puede realizar ya para comprender de mejor manera el texto audiovisual y, particularmente, las problemáticas que este presenta para la subtitulación. Debido a su carácter estratégico, el contenido de esta unidad se basa en las problemáticas más comunes de la subtitulación y en las maneras en que se pueden solucionar tomando en cuenta las restricciones y los propios recursos del filme. El trabajo de la unidad se lleva a cabo mediante la subtitulación de distintas secuencias de filmes y el continuo uso de programas informáticos especializados.

Cabe resaltar que ambas unidades toman como paradigma del texto audiovisual los productos cinematográficos de autor y de género; esto se debe a la explotación que los filmes hacen de los códigos y canales comunicativos, con fines diegéticos y extradiegéticos (Martínez-Sierra, 2012b), en cuanto promueven la participación de los estudiantes debido a sus afinidades a distintos géneros, directores u otros artistas.

La tercera unidad presenta el doblaje como una práctica profesional diferenciada por los mercados nacionales (o regionales). Asimismo, se estudian los rasgos que hacen de un doblaje un producto de calidad, mientras se revisan las convenciones más relevantes de la escritura de diálogos para la actuación voz. Durante la cuarta unidad se practica la producción de diálogos traducidos para el doblaje mediante la presentación de una serie de problemáticas usuales en distintos productos audiovisuales. Esta cuarta unidad, además de dedicarse al doblaje interlingüístico cotidiano, presentará otras modalidades relacionadas, como las voces superpuestas o la audiodescripción.

Por sus contenidos y actividades, el curso TR160 no busca ser un taller de especialización, sino que constituye una introducción al ámbito de la traducción audiovisual mediante sus dos modalidades más reconocidas - se trata del doblaje y la subtitulación, precisamente-.

Materiales y recursos. Los talleres que desarrollan la competencia de Traductología cuentan con dos documentos básicos: la guía del estudiante y el cronograma de trabajo (de forma independiente al sílabo académico que tiene un carácter oficial para toda la universidad). La guía del estudiante reúne las indicaciones fundamentales del curso y busca ser un documento accesible para el estudiante. En ese sentido, el documento contiene las fechas de entrega de trabajos, las consignas de las tareas y las rúbricas de calificación de los distintos trabajos (las rúbricas se mencionarán con mayor detalle más adelante). El cronograma de trabajo es un documento de consulta de los docentes del curso y no está disponible para los alumnos; contiene notas

Tabla 3

Secuenciación de temas de la segunda unidad

NÚMERO DE SESIÓN

8

9

10

11

12

13

14

\section{TEMAS DE LA SESIÓN}

El discurso guionado

El ruido y la redundancia semiótica

La intertextualidad

Los referentes culturales

La funciones del diálogo

El discurso especializado

Los juegos de palabras

Las canciones en películas musicales 
de coordinación y el resumen de los temas por sesión. En cuanto a los documentos, se incluye la bibliografía digitalizada del curso; estas lecturas son extractos de distintos libros relacionados con cinematografía y traducción audiovisual que los estudiantes revisan semanalmente. Asimismo, los estudiantes tienen a su disposición las diapositivas de las exposiciones en clase.

El material vinculado con las tareas de subtitulación de la segunda unidad incluye los videos de escenas de películas, los encargos de traducción, los guiones de las películas y los protocolos de subtitulación. Las escenas de películas fueron extraídas de la colección de archivos disponibles en la mediateca de la universidad y se tuvieron que seleccionar en un periodo de tres meses previos al inicio de clases. El proceso de selección de escenas partió de la cultura cinematográfica de los docentes, la búsqueda de las películas, la transformación y la extracción de las escenas en formatos editables y que fuesen fáciles de compartir por las plataformas educativas disponibles. Los encargos de subtitulación partieron de situaciones reales a las que se han enfrentado los docentes del curso y la elaboración de órdenes de servicio, en las que se incluían datos como el tipo de archivo que el cliente entregaba, los servicios de valor añadido requeridos, las tarifas por los servicios, además de las coordenadas usuales de otras modalidades de traducción, como los plazos de entrega, información del cliente, entre otros. Puede consultarse un ejemplo de encargo en la Figura 1.

Los guiones de las películas proceden de bases de datos disponibles en internet y se seleccionan solamente las páginas puntuales para la consulta de los estudiantes. Debido a que durante la primera edición del curso no se podía garantizar el uso de solo guiones de posproducción, fue tarea de los estudiantes comprobar de qué tipo de guiones se trataba. Este fue un ejercicio adicional que resultó provechoso para garantizar que los estudiantes prestaran más atención a los textos fuente. Por otro lado, con base en las pautas de Díaz-Cintas y Remael (2007), se entrega un protocolo de subtitulación cada semana (por cuatro semanas). Cada protocolo reúne las pautas específicas que los estudiantes necesitan seguir para subtitular las escenas de las tareas semanales puede consultarse un ejemplo de protocolo en la Tabla 4-. El razonamiento para segmentar los protocolos en cuatro es que los estudiantes pueden olvidar las pautas pertinentes para la subtitulación si es que reciben un protocolo extenso. En cambio, en la medida que reciben las pautas de manera secuenciada y de acuerdo con las problemáticas que se estudian cada semana, tienen más oportunidades para prestar atención a las pautas pertinentes.

En la primera edición del curso se utilizaron cuatro plataformas de comunicación y de almacenamiento de información. La más importante fue el Aula Virtual (Blackboard), que se actualizaba semanalmente con los contenidos de las sesiones. Además, esta plataforma sirvió para la entrega de todas las tareas programadas en el curso. Asimismo se utilizó, en un primer momento, la plataforma Dropbox para cargar los videos para subtitular y poder luego compartir los vínculos de descarga con los estudiantes. Luego se migró todo el contenido a la plataforma de almacenamiento OneDrive debido a la capacidad de almacenamiento que esta ofrecía. Se utilizó también el correo institucional para mantener el contacto con los estudiantes y enviarles los vínculos de descarga de los videos de acuerdo con una dinámica real de comunicación con el cliente.

El curso se lleva a cabo en laboratorios equipados con audífonos. Se utilizan solo programas de licencia libre, como Subtitle Workshop y Aegisub para la subtitulación; con el programa aTube Catcher se transforman los formatos de video y con el programa AviRecomp se hace la incrustación de subtítulos en la imagen. Se trabaja solo con el formato genérico de subtítulos SubRip (.srt). Los estudiantes, por su parte, deben contar con estos programas en sus computadoras personales. Todos estos son los recursos utilizados en las dos unidades vinculadas con la subtitulación.

Metodología. Las clases se desarrollan de acuerdo con una metodología activa; el alumno aplica las estrategias propuestas en clase y elabora productos que evidencien su aprendizaje mediante la articulación de contenidos de tipo teórico y las experiencias que acumule. Son dos sesiones semanales, cada una con una duración de 180 minutos. Las sesiones cuentan con partes introductorias sobre los temas de la unidad y ejercicios de aplicación tanto dentro como fuera del aula. En el caso de las sesiones de la primera unidad, los 
SEMANA 5

$5 \%$ DE LA CD2

- El estudiante debe realizar la subtitulación interlingüística de la escena 27 de la película Dear White People ISIMIEN 2014). Los subtítulos deben adecuarse a las pautas del «Protocolo B».

- En esta oportunidad se considerará la cantidad de caracteres predeterminada por Subtitle Workshop.

- De forma previa a la subtitulación, el estudiante debe redactar una sinopsis de la película (150 a 200 palabras) y el análisis de la escena (250 a 300 palabras), que también se incluirán como productos de esta entrega.

- La entrega del trabajo se realizará mediante Blackboard, en una carpeta comprimida que solo debe contener dos archivos: un documento de Word con la sinopsis, el análisis y el comentario, y el de los subtítulos en formato SubRip (.srt).

\section{PAUTADO DEL ENCARGO DE TRADUCCIÓN}

\section{General}

Distribuidora: Delta Films SAC

Exhibición: No comercial (festival de cine en mayo, Centro Cultural Ricardo Palma)

Caracteres por línea: No se especifica

Protocolo de la distribuidora: No incluido

Transferencia: Descarga directa por vínculo web

Archivo de película: Copia digital, formato AVI (sin marcas de agua; sin protección de copiado)

Derechos de traducción: No asignados al traductor ni solicitados por la distribuidora

Tarifa por palabra: PEN 45 por cada 180 palabras

Dialogue list: No

Guion de preproducción o posproducción: Sí (pero por comprobar)

\section{Servicios requeridos}

Transcripción de diálogos: No

Traducción: Sí

Segmentación y ajuste: Sí

Localización: Sí

\section{Tarifa}

Traducción: PEN 35 por 180 palabras

Segmentación, ajuste y localización: PEN 12 por minuto

Solo segmentación y ajuste: PEN 8 por minuto

Solo localización: PEN 8 por minuto

Transcripción: PEN 8 por minuto

\section{Datos de entrega al cliente}

Subtítulos sin incrustar

Archivo en formato de subtítulos general (preferiblemente .srt) o sin etiquetas

Por correo electrónico

19 de septiembre, antes de las 22:00

Figura 1. Ficha de tarea y pautado de subtitulación 
Tabla 4

Modelo de protocolo de subtitulación

\section{PROTOCOLO B}

\begin{tabular}{|c|c|c|}
\hline \multicolumn{3}{|l|}{ Ortotipografía } \\
\hline & $\begin{array}{l}\text { Fuente } \\
\text { Tamaño }\end{array}$ & $\begin{array}{l}\text { (palo seco) Arial, Helvética } \\
14 \text { puntos }\end{array}$ \\
\hline \multicolumn{3}{|l|}{ Formato } \\
\hline & $\begin{array}{l}\text { Fuente } \\
\text { Color } \\
\text { Alineación }\end{array}$ & $\begin{array}{l}\text { con contorno o sombra } \\
\text { blanco o amarillo } \\
\text { texto centrado }\end{array}$ \\
\hline \multicolumn{3}{|l|}{ Extensión } \\
\hline & $\begin{array}{l}\text { Caracteres } \\
\text { Líneas }\end{array}$ & $\begin{array}{l}37 \text { (TV), } 35 \text { (VHS), } 40 \text { (DVD), } 43 \text { (IND.) } \\
\text { incluyen espacios en blanco y signos de puntuación } \\
\text { máximo } 2 \text { líneas } \\
\text { línea superior de igual extensión que la inferior } \\
\text { línea superior de menor extensión que la inferior }\end{array}$ \\
\hline \multicolumn{3}{|l|}{ Duración } \\
\hline & $\begin{array}{l}\text { Mínima } \\
\text { Máxima } \\
\text { Cambio de toma } \\
\text { Pausa entre subtítulos }\end{array}$ & $\begin{array}{l}1 \text { segundo } \\
6 \text { segundos } \\
\text { El subtítulo desaparece. } \\
\text { Se incluye una breve pausa entre subtítulos consecutivos. }\end{array}$ \\
\hline \multicolumn{3}{|l|}{ Sintaxis } \\
\hline & Segmentación & No se separan sintagmas o unidades de sentido. \\
\hline \multicolumn{3}{|l|}{ Puntuación } \\
\hline & $\begin{array}{l}\text { Coma } \\
\text { (,) }\end{array}$ & $\begin{array}{l}\text { No tiene un uso regular con respecto a la normativa del español. } \\
\text { Su uso se privilegia cuando existe la posibilidad de falsos sentidos } \\
\text { o para imitar la prosodia del diálogo. } \\
\text { Se evita el uso de comas al final de un subtítulo para evitar } \\
\text { confusiones con el punto final. }\end{array}$ \\
\hline
\end{tabular}

Nota: Adaptado de Audiovisual Translation: Subtitling (pp. 80-143), por J. Díaz-Cintas y A. Remael, 2007, Manchester: Saint Jerome Publishing. Copyright 2007 por Jorge Díaz Cintas y Aline Remael.

estudiantes desarrollan ejercicios en cada sesión que varían en su dificultad y que incluyen progresivamente las habilidades adquiridas en sesiones anteriores.

Las sesiones de la segunda unidad tienen una estructura similar. Se parte de actividades de motivación para luego pasar a la parte de exhibición de escenas con problemáticas particulares. Posteriormente las problemáticas se analizan y se proponen formas de resolución de acuerdo con la creatividad de los estudiantes y dentro del marco de las restricciones propias de la subtitulación y los protocolos de subtitulación. En una segunda parte de las sesiones, los estudiantes tienen tiempo para subtitular en aula y hacer las consultas necesarias a los docentes mientras se familiarizan con los programas informáticos. Antes de finalizar la sesión, un estudiante presenta sus subtítulos y en ese proceso tanto estudiantes como docentes brindan retroalimentación. Los estudiantes reciben un encargo de traducción fuera de aula; uno de ellos deberá presentar la subtitulación al inicio de la siguiente sesión. En las últimas semanas de la segunda unidad, los estudiantes realizan exposiciones grupales sobre escenas que les fueron encargadas para subtitular. En estos casos, el proceso de subtitulación en aula se suprime, pero los alumnos aún tenían que llevar una escena para subtitular fuera de aula. 
La metodología utilizada permitió abarcar las prioridades que señalan Batrina y Espasa (2005): legibilidad, oralidad y síntesis; a su vez, estas prioridades sirvieron como criterios de calidad que debían tenerse en cuenta para la evaluación de los productos $y$, consecuentemente, se incluyeron en la rúbrica de evaluación (véase más adelante y en el Apéndice 1). Asimismo, se practicaron, tanto dentro como fuera de aula, la serie de tareas básicas que señala esta autora, a saber, el visionado, la localización, la traducción y la edición.

Evaluación. Las evaluaciones son continuas y corresponden a la presentación de distintos productos o evidencias de aprendizaje. Estos productos evaluados se desarrollan fuera de aula; este trabajo se calcula en, aproximadamente, tres horas semanales. Las consignas de las evaluaciones se encuentran establecidas en la guía del estudiante, mediante fichas de trabajo del siguiente tipo.

Las evaluaciones de las dos primeras unidades incluyeron la elaboración de los productos mencionados en la Tabla 5 que se presenta a continuación.

Como se mencionó antes, se elaboraron rúbricas para la calificación de los trabajos. Estas rúbricas son de tipo cualitativo a pesar de que cuentan con algunos descriptores de tipo cuantitativo. La razón de las rúbricas se vincula con el modelo de evaluación formativa, dado que los estudiantes reciben sus trabajos calificados con las rúbricas debidamente anotadas a modo de retroalimentación. Este relieve de la rúbrica como instrumento de evaluación y calificación se basa en el interés particular de la Traductología -y en general de las competencias comunicativas en lenguas extranjeras- en establecer modelos de competencias y constructos de evaluación apropiados para medir el desempeño del traductor, así como para ponderar la calidad del producto, como demuestra Waddington (2000) en su exhaustivo estado de la cuestión sobre este tema. Se puede revisar la rúbrica de evaluación de subtítulos en el Apéndice 1.

Esta rúbrica se basó en una herramienta de calificación previa, denominada «Rúbrica de la traducción como proceso» que se utiliza en los talleres de traducción de los niveles quinto y sexto; no obstante, las dimensiones y descriptores de los niveles de logro guardan relación con aquellos que Sponholz (2003) reúne en su estado del arte y modelo de evaluación -aunque esta autora no propone un instrumento aplicado propiamente dicho-. Se optó por no utilizar un baremo negativo laquel que resta puntos por error), debido a que se trata de los primeros ejercicios de subtitulación de los estudiantes y se prefiere evitar algún tipo de desmotivación en los alumnos. Asimismo, la idea de trabajar con un modelo «global» de rúbrica, aunque enfocada en la subtitulación, se relaciona con la crítica de Waddington (2000) sobre el enfoque de suma de errores.

\section{CONCLUSIÓN}

El curso de Doblaje y Subtitulación (TR160) de la carrera de Traducción e Interpretación Profesional representa un alcance didáctico que comparte los objetivos de

\section{Tabla 5}

Lista de actividades fuera de aula

SEMANA

1

2

3

4

5

6

7
TAREA

Línea del tiempo de la traducción audiovisual

Glosario audiovisual de términos cinematográficos

Informe sobre el visionado y contraste de dos productos audiovisuales subtitulados

Escena subtitulada (trabajo individual)

Exposición grupal 1

Exposición grupal 2

Exposición grupal 3

Escena subtitulada e incrustación de subtítulos (evaluación de medio semestre) 
trabajos previos (Díaz \& Orero, 2003; Sponholz, 2003; Díaz \& Remael, 2007). Sin embargo, por su naturaleza curricular, se trata de una propuesta enfocada en la aplicación inmediata por parte de los docentes como sucede en el caso de la propuesta de MartínezSierra (2012c)-. Son características relevantes del curso, el énfasis en la articulación con un modelo de competencia traductora integral (TIP-UPC), así como en los fundamentos teóricos que sustentan la concepción de la traducción (perspectiva funcional, normativa y semiótico). Asimismo, resulta relevante el diseño de evaluaciones (tareas y rúbricas) y de materiales de enseñanza (protocolos de subtitulación, fichas de trabajo fuera de aula y guías del alumno). Estas tres características permiten que el curso se articule con las dimensiones y niveles de logro establecidos en la competencia específica de Traductología; de ello deriva su importancia.

Con respecto a su primera edición, el curso abordó de manera efectiva los contenidos básicos para la inducción a la traducción audiovisual y, de forma particular, a la subtitulación en sus dos primeras unidades temáticas. En este sentido, los logros planteados a priori a nivel general y específico fueron alcanzados. En la ejecución del curso, se pudo apreciar una motivación intrínseca en los estudiantes - aquello que Kelly (2005, p. 49) define como el hecho de «fulfilling personal potential [...] gaining knowledge for its own sake, or furthering a potential interest»-, el tema del cine y la conceptualización de los productos audiovisuales como textos les permitió aproximarse a un pasatiempo desde una perspectiva más especializada y analítica. De ahí que cumplieran con las lecturas y actividades dentro y fuera de aula con una actitud proactiva. Este hecho facilitó el dictado del curso en su primera edición; sin embargo, en siguientes ediciones esta actitud varió de acuerdo con el grupo de alumnos. A partir de la retroalimentación de los propios estudiantes y las evaluaciones docentes que se aplicaron poco antes de la evaluación parcial, se puede afirmar que sus expectativas fueron alcanzadas.

En el marco de la secuenciación de los contenidos, haber apostado por una primera unidad de carácter teórico-analítico sirvió de mucho, dado que brindó a los estudiantes las herramientas que luego les sirvieron para comprender el texto audiovisual en sus diferentes canales de transmisión y códigos. Asimismo, esto permitió que no se enfocaran solo en los aspectos lingüísticos. En ese sentido, la comprensión del producto audiovisual como texto tuvo implicaciones positivas en la manera en que los estudiantes planteaban la resolución de problemas de traducción. Ello se pudo evidenciar en la presentación de las evaluaciones que incluían subtitulación y comentarios reflexivos sobre las estrategias de traducción que utilizaron para los focos de dificultad de las escenas asignadas.

En otro sentido, también existe una perspectiva autocrítica sobre algunos aspectos del diseño del curso y que han debido modificarse para su segunda y tercera edición. Entre algunos temas puntuales se encuentran la reasignación de tiempos a algunos contenidos vinculados con el análisis de escenas durante la primera unidad; dado que se ha tratado de reducir el tiempo de las exposiciones teóricas para propiciar la aplicación de análisis más inductivos. Por otro lado, también fue necesario dedicar tiempo exclusivo al acompañamiento de los estudiantes en aula mientras se enfrentaban por primera vez a los programas informáticos de subtitulación. Se consideró, además, el diseño de materiales instructivos para el uso de estas herramientas (manuales de uso de Subtitle Workshop y Aegisub). Se buscó garantizar que los estudiantes ahorraran esfuerzos en dominar dichos programas informáticos y así puedan enfocarse en las demás restricciones propias de la subtitulación.

Finalmente, se pueden mencionar algunos retos que el programa académico y los docentes tendrán que enfrentar en el mediano plazo. Se deben promover las salidas profesionales vinculadas con la subtitulación. Esto implica establecer vínculos entre instituciones privadas y públicas y la universidad para que los estudiantes puedan realizar prácticas preprofesionales. Este no es solo un reto pedagógico, sino también parte del compromiso de profesionalización de la traducción y la interpretación que el programa profesional ha asumido. Desde esta perspectiva, la oferta de servicios especializados de subtitulación que se mencionó páginas antes tendría que partir de la propia universidad, dado que el gremio profesional actual carece de estos conocimientos. 
La migración del curso a una modalidad semipresencial o con un componente blended es otro reto de diseño instruccional que todo el programa académico TIP-UPC deberá enfrentar en el proceso hacia el 2019. Sobre este tipo de diseños existen realmente pocas experiencias registradas (Dorado \& Orero, 2007); el equipo docente espera, por ello, poder colaborar con las experiencias que logren acumularse en los siguientes años.

\section{REFERENCIAS}

Batrina, F., \& Espasa, E. (2005). Audiovisual Translation. En M. Tennent (Ed.), Training for the New Millennium. Pedagogies for translation and interpreting (pp. 83-100). Amsterdam y Philadelphia: John Benjamins.

Cerezo, B. (2013). La formación en Traducción Audiovisual en España: Un estudio de caso empírico descriptivo. Trans, 17, 167-183.

Díaz-Cintas, J., \& Orero, P. (2003). Postgraduate Courses in Audiovisual Translation. The Translator, 9(2), 371-388. doi: 10.1080/13556509.2003.10799164

Diaz-Cintas, J., \& Remael, A. (2007). Audiovisual Translation: Subtitling. Manchester: Saint Jerome Publishing.

Dorado, C., \& Orero, P. (2007). Teaching Audiovisual Translation Online: A Partial Achievement. Perspectives: Studies in Translatology, 15 (3), 191-202. doi: 10.1080/13670050802153988

El Comercio. (2014). Cartelera. Lima, Perú: Grupo Editorial El Comercio. Recuperado de http://elcomercio.pe/cartelera.

Granell, X. (2011). Teaching Video Game Localisation in Audiovisual Translation courses at university. The Journal of Specialised Translation, 16, 185-202.

Kelly, D. (2005). A Handbook for Translator Trainers. A Guide to Reflective Practice. Manchester: Saint Jerome Publishing.

Lachat, C. (2011). Didáctica de la traducción audiovisual: enseñar a mirar. Revista Electrónica de Didáctica de la Traducción y la Interpretación, 6, 94-105.

Martínez-Sierra, J. J., Ed. (2012a). Reflexiones Sobre La Traducción Audiovisual. Tres Espectros, Tres Momentos. Valencia: Universitat de Valencia.

Martínez-Sierra, J. J. (2012b). On the Relevance of Script Writing. Basics in Audiovisual Translation Practice and Training. Cadernos de Tradução,29(1),145-163. doi:10.5007/2175-7968.2012v1n29p145

Martínez-Sierra, J. J. (2012c). Introducción a la traducción audiovisual. Murcia: Editum.

Nord, C. (2005). Text Analysis in Translation. Theory, Methodology, and Didactic Application of a Model for Translation-Oriented Text Analysis. Amsterdam: Rodopi.
Nord, C. (2014) Translating as a Purposeful Activity. Functuonalist Approaches Explained. London y New York: Routledge.

Remael, A. (2004). A Place for Film Dialogue Analysis in Subtitling Courses. En P. Orero (Ed.), Topics in Audiovisual Translation. (pp. 103-126). Amsterdam y Philadelphia: John Benjamins Publishing Company. doi: 10.1075/btl.56.13rem

Sponholz, C. (2003). Teaching Audiovisual Translation Theoretical Aspects, Market Requirements, University Training and Curriculum Development (Tesis de Grado). Johannes Gutenberg-Universität Mainz, Mainz, Alemania.

Toury, G. (2004). Los Estudios Descriptivos de Traducción y más allá. Metodología de la investigación en Estudios de Traducción. Madrid: Cátedra.

Waddington, C. (2000). Estudio comparativo de diferentes métodos de evaluación de traducción general. Madrid: Universidad Pontificia Comillas. 


\section{APÉNDICE 1}

Rúbrica de evaluación de los subtítulos

\begin{tabular}{|c|c|c|c|c|}
\hline CRITERIO & MUY BUENO & BUENO & REGULAR & INSUFICIENTE \\
\hline $\begin{array}{l}\text { Sincronía y } \\
\text { segmentación } \\
5 \text { puntos }\end{array}$ & $\begin{array}{l}\text { Ajusta del } 100 \text { al } 95 \% \\
\text { de subtítulos de manera } \\
\text { exacta tomando en } \\
\text { consideración los periodos } \\
\text { de enunciación en la } \\
\text { escena. } \\
\text { Segmenta del } 100 \text { al } 95 \\
\% \text { de los enunciados de } \\
\text { manera adecuada tomando } \\
\text { en cuenta los principios } \\
\text { de los } 6 \text { y } 1 \text { segundo y las } \\
\text { características de la acción } \\
\text { en pantalla. } \\
5 \text { puntos }\end{array}$ & $\begin{array}{l}\text { Ajusta del } 95 \text { al } 85 \% \text { de } \\
\text { subtítulos de manera } \\
\text { exacta. } \\
\text { Segmenta del } 95 \% \text { al } \\
85 \% \text { de los enunciados } \\
\text { de manera adecuada } \\
\text { tomando en cuenta } \\
\text { los principios de los } \\
6 \text { y } 1 \text { segundo y las } \\
\text { características de la } \\
\text { acción en pantalla. } \\
4 \text { puntos }\end{array}$ & $\begin{array}{l}\text { Ajusta del } 85 \text { al } 75 \% \text { de } \\
\text { subtítulos de manera } \\
\text { exacta. } \\
\text { Segmenta del } 85 \% \text { al } \\
75 \% \text { de los enunciados } \\
\text { de manera adecuada } \\
\text { tomando en cuenta los } \\
\text { principios de los } 6 \text { y } 1 \\
\text { segundo. } \\
3 \text { a } 2 \text { puntos }\end{array}$ & $\begin{array}{l}\text { Ajusta de manera } \\
\text { inadecuada más de } 25 \\
\% \text { de los subtítulos. } \\
\text { Segmenta de manera } \\
\text { inadecuada más del } 25 \\
\% \text { de los subtítulos. } \\
1 \text { punto }\end{array}$ \\
\hline $\begin{array}{l}\text { Adecuación al } \\
\text { protocolo y al } \\
\text { encargo } \\
5 \text { puntos }\end{array}$ & $\begin{array}{l}\text { Cumple cabalmente con la } \\
\text { normativa del protocolo de } \\
\text { subtitulación (corrección } \\
\text { ortográfica, ortotipográfica, } \\
\text { cantidad de caracteres) } \\
\text { que es parte del encargo. } \\
\text { Produce un TM de acuerdo } \\
\text { a las especificaciones del } \\
\text { translation brief prestando } \\
\text { especial atención a tener } \\
\text { un producto de excelente } \\
\text { calidad. } \\
5 \text { puntos }\end{array}$ & $\begin{array}{l}\text { Cumple con las pautas } \\
\text { del protocolo salvo en } \\
\text { el caso de algunos } 11 \text { o } \\
\text { 2) errores relacionados } \\
\text { con la ortografía, la } \\
\text { ortotipografía o la } \\
\text { cantidad de caracteres. } \\
\text { Traduce el TF de } \\
\text { manera exhaustiva de } \\
\text { acuerdo al translation } \\
\text { brief. } \\
4 \text { puntos }\end{array}$ & $\begin{array}{l}\text { Cumple con las pautas } \\
\text { del protocolo salvo en } \\
\text { el caso de algunos } 13 \text { o } \\
\text { 4) errores relacionados } \\
\text { con la ortografía, la } \\
\text { ortotipografía o la } \\
\text { cantidad de caracteres. } \\
3 \text { a } 2 \text { puntos }\end{array}$ & $\begin{array}{l}\text { Comete } 5 \text { o más } \\
\text { errores relacionados } \\
\text { con la normativa de } \\
\text { los protocolos de } \\
\text { subtitulación. } \\
\text { No traduce el TF en su } \\
\text { totalidad de injustificada } \\
\text { o debido a falta de } \\
\text { comprensión. } \\
1 \text { punto }\end{array}$ \\
\hline $\begin{array}{l}\text { Traducción y } \\
\text { síntesis } \\
5 \text { puntos }\end{array}$ & $\begin{array}{l}\text { Reexpresa el sentido } \\
\text { del TF en sus distintos } \\
\text { segmentos de manera } \\
\text { correcta sin ningún caso } \\
\text { de sobretraducción o } \\
\text { subtraducción. } \\
\text { Reproduce todos } \\
\text { los matices del TF } \\
\text { (semióticos, pragmáticos y } \\
\text { comunicativos) de acuerdo } \\
\text { a la situación comunicativa } \\
\text { receptora y al género } \\
\text { audiovisual del TF. } \\
5 \text { puntos }\end{array}$ & $\begin{array}{l}\text { Vierte el sentido general } \\
\text { de TF de manera } \\
\text { consistente realizando } \\
\text { la síntesis propia } \\
\text { de la subtitulación } \\
\text { sin producir falsos } \\
\text { sentidos. } \\
\text { Reexpresa la mayoría } \\
\text { de matices del texto } \\
\text { fuente de manera } \\
\text { efectiva. } \\
4 \text { puntos }\end{array}$ & $\begin{array}{l}\text { Mantiene el sentido } \\
\text { del TF a pesar de que } \\
\text { no llega a reproducir } \\
\text { algunos matices o } \\
\text { comete algunos errores } \\
\text { de sentido ( } 2 \text { o menos), } \\
\text { salvo contrasentidos. } \\
3 \text { a } 2 \text { puntos }\end{array}$ & $\begin{array}{l}\text { Comete varios ( } 3 \circ+\text { ) } \\
\text { errores de sentido (falso } \\
\text { sentido, contrasentido, } \\
\text { sinsentido) debido a la } \\
\text { falta de comprensión } \\
\text { del TF o una inadecuada } \\
\text { síntesis de los } \\
\text { segmentos. } \\
\text { No traduce el TF en su } \\
\text { totalidad de manera } \\
\text { premeditada o debido a } \\
\text { falta de comprensión. } \\
1 \text { punto }\end{array}$ \\
\hline $\begin{array}{l}\text { Estrategias de } \\
\text { traducción y } \\
\text { creatividad } \\
5 \text { puntos }\end{array}$ & $\begin{array}{l}\text { Mantiene la consistencia } \\
\text { de sus decisiones } \\
\text { traductoras a lo largo } \\
\text { del TM. } \\
\text { Identifica problemas } \\
\text { de traducción y toma } \\
\text { decisiones traductoras } \\
\text { apropiadas según el TF y el } \\
\text { translation brief en todos } \\
\text { los casos (100-90\%). } \\
5 \text { puntos }\end{array}$ & $\begin{array}{l}\text { Toma decisiones } \\
\text { traductoras apropiadas } \\
\text { según el TF y el } \\
\text { translation brief para } \\
\text { resolver problemas } \\
\text { de traducción en la } \\
\text { mayoría de los casos } \\
\text { (90-70\%). } \\
4 \text { puntos }\end{array}$ & $\begin{array}{l}\text { Identifica y } \\
\text { soluciona algunos } \\
\text { de los problemas de } \\
\text { traducción (60-50\%) } \\
\text { Toma algunas } \\
\text { decisiones (1 o 2) que } \\
\text { conllevan a errores de } \\
\text { traducción. } \\
3 \text { a } 2 \text { puntos }\end{array}$ & $\begin{array}{l}\text { Toma varias decisiones } \\
(3 \text { o }+) \text { que conllevan a } \\
\text { errores de traducción. } \\
\text { No concluye la } \\
\text { traducción debido a } \\
\text { la imposibilidad de } \\
\text { resolver los problemas } \\
\text { de traducción. } \\
1 \text { punto }\end{array}$ \\
\hline
\end{tabular}




\section{NOTAS}

1. Los programas de formación de traductores en el Perú se ubican en el nivel de educación superior técnica (3 años) y universitaria ( 5 años). Luego de culminar la formación profesional, los egresados de los programas universitarios acceden al grado académico de bachilleres. Por pregrado, se entiende el conjunto de cursos y niveles que conducen al grado de bachiller. Los estudios de posgrado incluyen los programas de maestría y doctorado, y otras especializaciones que estén reguladas por instituciones universitarias y registren créditos académicos.

2. Entre los festivales más representativos en el Perú, se puede mencionar el Festival de Lima de la Pontificia Universidad Católica, Al Este de Lima, Lima Independiente y el Outfest Perú. De estos, los dos primeros cuentan con auspicio de empresas subtituladoras europeas; sin embargo, en el caso de los otros dos festivales, se recurre también a servicios locales para realizar la subtitulación (urgente) de algunas de las piezas que toca exhibirse.

3. En el marco de las esferas privadas, también se puede mencionar que existe una amplia cantidad de aficionados a los productos audiovisuales de Japón y Corea del Sur. Estos aficionados producen, como sucede en muchos otros espacios, subtítulos para su propia comunidad de práctica. Si bien los productos subtitulados circulan por la web sin fines de lucro, una de las primeras iniciativas de subtitulación por aficionados y para aficionados se inició en la década de 1990 en Lima, con la publicación Sugoi y su línea de cintas de video denominadas AfiSubs.

4. En el Perú, el título profesional tiene el carácter de una certificación a nombre de la Nación que permite el ejercicio de determinados profesionales. En el caso del ejercicio profesional de la traducción y la interpretaciòn, no está regulado por el Estado para garantizar la exclusividad de los profesionales con formación universitaria de especialidad en estos campos.
5. Es conocido que la reforma educativa europea establece un enfoque por competencias en los distintos niveles de formación; lo que ha conllevado grandes cambios en la metodología didáctica tanto escolar como universitaria (Cerezo, 2013: 169). Sin embargo, los modelos por competencias son, hasta el momento en el Perú, una elección de cada institución académica. Se sabe que la nueva reforma de la educación superior asume el enfoque por competencias; no obstante, su implementación aún está pendiente.

6. No obstante, debe tomarse en cuenta que el desarrollo de las demás habilidades en paralelo es un elemento fundamental para conseguir el logro de niveles de la competencia de Traductología en particular. Se considera que un desarrollo óptimo de un programa basado en competencias debe contemplar asignaturas que integren dos o más habilidades específicas en los niveles más altos de desarrollo.

7. Resulta importante plantear esta característica del programa profesional, pues otros modelos de competencia traductora se basan en modelos descriptivos de perfiles profesionales existentes. Este es el caso del modelo de competencia traductora del grupo PACTE. Un planteamiento prospectivo de competencia traductora no solo concibe que el egresado de un programa profesional se inserte en el mercado laboral, sino que llegue a transformarlo. Dadas las condiciones de desarrollo de la profesión del traductor en el Perú, la formación de nuevas generaciones de profesionales de la traducción exige pensar en propuestas innovadoras y generadoras de cambio.

(c) Los autores. Este artículo es publicado por la Revista Digital de Investigación en Docencia Universitaria del Área de Institutional Research and Effectiveness de la Dirección de Aseguramiento de la Calidad, Universidad Peruana de Ciencias Aplicadas. Este es un artículo de acceso abierto, distribuido bajo los términos de la LicenciaCreativeCommons Atribución-Compartirlgual 4.0 Internacional.( http://creativecommons.org/licenses/by-sa/4.0/), que permite el uso no comercial, distribución y reproducción en cualquier medio, siempre que la obra original sea debidamente citada. 